\title{
Terapia asistida por perros en el tratamiento del manejo de las emociones en adolescentes
}

\section{Dog-assisted therapy to treat emotional management in teenagers}

\author{
Xóchitl Ortiz Jiménez $\quad$ René Landero Hernández Mónica González Ramírez \\ Universidad Autónoma de Nuevo León, México \\ (Rec: septiembre de 2012 - Acep: noviembre de 2012)
}

\begin{abstract}
Resumen
Los cambios emocionales en la adolescencia se relacionan con la dificultad para percibir, comprender y regular las emociones, lo que impacta negativamente sobre su autoestima y el manejo de las emociones. La terapia asistida por perros puede aumentar la autoestima de los adolescentes y ayudarlos a mejorar en el manejo de las emociones. El objetivo de este estudio fue analizar los efectos de la terapia asistida por perros sobre la autoestima y los componentes de las emociones en un grupo de adolescentes con problemas emocionales. Los resultados mostraron cambios significativos en la atención emocional de los adolescentes, es decir, en su capacidad para percibir mejor sus emociones.
\end{abstract}

Palabras clave: terapia asistida por animales, emociones y adolescentes.

\begin{abstract}
The emotional changes in adolescence are related to the difficulty to perceive, understand and regulate emotions, which negatively impacts on self-esteem and the management of emotions. Dog-assisted therapy can help improve self-esteem and help in the management of emotions. The aim of this study was to analyze the effects of dog-assisted therapy on self-esteem and components of emotions in a group of adolescents with emotional problems. The results showed significant changes in the attentional emotion component of emotions, which means, in the ability to perceive their emotions.
\end{abstract}

Keywords: animal assisted therapy, emotions and adolescents.

\footnotetext{
Correspondencia dirigida a: Facultad de Psicología, Universidad Autónoma de Nuevo León, Mutualismo 110, Col. Mitras Centro, Monterrey, NL, 64460, México. Tel: +52-81-8348-3866. Fax: +52-81-8333-7859. E-mail: xortizj@ gmail.com
} 


\section{Introducción}

El interés en la literatura científica por conocer cómo los animales afectan física y psicológicamente a las personas que conviven con ellos ha crecido recientemente. Algunos estudios interesados en conocer los efectos sobre la salud han demostrado valores más bajos de estrés y más altos en salud mental en las personas que conviven con perros como animales de compañía y que los consideran como miembros de la familia (Alonso, 2000; González \& Landero, 2011). Lo anterior indica que los animales producen efectos positivos sobre la salud física y mental de las personas que conviven con ellos (Alonso, 2000; Gómez, Atehortua \& Orozco, 2007). Otros estudios han demostrado que los profesionales de la salud que se apoyan en animales como coterapeutas en el desarrollo de su trabajo terapéutico obtienen cambios positivos en sus pacientes. A esta intervención se le conoce como Terapia Asistida por Animales (TAA), la cual se define como una intervención diseñada para mejorar el funcionamiento cognitivo, físico, social y emocional de un paciente, con objetivos específicos y delimitados en el tiempo (Villalta \& Ochoa, 2007).

La TAA se utiliza en diversas poblaciones como niños con autismo, adolescentes en situaciones de riesgo, adultos mayores con demencia, personas con alteraciones neuropsicológicas, con trastornos psiquiátricos como esquizofrenia y con discapacidad física. En todos los estudios se reporta una mejoría en el funcionamiento social-interpersonal, así como en la ejecución de las actividades de la vida diaria y el bienestar general (Anzizu, Botella \& Ferrer, 2001; Fuentes, Gallego, Baza \& Martín, 2012; Vázquez \& Lista, 2011). Lo anterior sugiere que la participación de los animales como coterapeutas puede ser de utilidad para el tratamiento de diversas enfermedades o problemas psicológicos relacionados con las emociones, como ansiedad, estrés, depresión y baja autoestima. La autoestima se refiere a la confianza que tiene la persona en sí misma, pudiendo tener una orientación positiva o negativa en la valoración que hace de las cogniciones (Pearlin, 1989; Rosenberg, 1989), y el grado de satisfacción personal se expresa en el comportamiento de la persona (Guindon, 2002). Hay varios factores que impactan en la autoestima de las personas, uno de ellos es el manejo de las emociones, ya que las emociones además de implicar la percepción subjetiva de la persona que la experimenta (evaluación cognitiva), incluyen la suposición o percepción que los otros hacen de esa emoción y esa valoración de los otros influye en la autoestima de la persona que experimenta la emoción.
Por ello, en las interacciones sociales las expresiones emocionales verbales y no verbales transmiten información acerca de los propios pensamientos, de las intenciones y conductas (Keltner \& Haidt, 2001).

El adecuado manejo de las emociones o competencia emocional incluye la habilidad para percibir, usar, comprender y regular las propias emociones y las de los demás, para un funcionamiento social óptimo (Denham et al., 2003; Eisenberg, Fabes, Guthrie, \& Reiser, 2000; Savage, 2002). Por ejemplo, la habilidad para percibir con precisión el tipo e intensidad de emoción de una persona, facilita la predicción y comprensión de las acciones posteriores de esa persona (Elfenbein, Marsh, \& Ambady, 2002). La comprensión de los estados emocionales respecto a la relación ambientepersona guía la atención, la toma de decisiones y las respuestas conductuales (Damasio, 1994). El manejo adecuado de las emociones es fundamental para un óptimo funcionamiento social, ya que permite tanto la expresión de las emociones como de la conducta socialmente apropiada. Actualmente, se conoce que las emociones incluyen los siguientes componentes: atención emocional, claridad emocional y regulación emocional (Fernández-Berrocal, Extremero \& Ramos, 2004).

Las cambios emocionales forman parte de la vida humana y pueden presentarse en diversas etapas de la vida. Una de las etapas más susceptibles es la adolescencia, una fase crucial y de cambio en el desarrollo del ser humano, ya que en ella se establece la identidad, la competencia social, el ajuste emocional y la resiliencia psicológica (Herbert, 2008). La adolescencia incluye a los jóvenes de los 12 hasta los 18 años de edad, quienes experimentan diversos cambios que impactan todos los aspectos de su vida y que incluyen el crecimiento físico, las cogniciones, las emociones y el funcionamiento social (Herbert, 2008). En el aspecto emocional, la adolescencia significa la capacidad de sentir, desarrollar y manifestar las emociones. Esta capacidad de experimentar el mundo emocional trae consigo tanto emociones positivas como negativas, de tal manera que el adolescente puede sentir tristeza, alegría, miedo, ansiedad, estrés, entre otras. Sin embargo, esta capacidad de experimentar diversas emociones puede afectar la vida social y personal del adolescente debido a la dificultad para manejarlas adecuadamente (Keltner, Moffit \& Stouthamer-Loeber, 1995).

En relación con las terapias o intervenciones, es muy raro que los adolescentes las busquen por ellos mismos. Generalmente son referidos por sus padres ,quienes se preocupan por su desempeño académico 
o su comportamiento en la escuela o en casa. No obstante, aunque acepten participar en alguna terapia, les es difícil permanecer en ella por largos periodos y tienden a ver su vida y sus problemas de forma diferente a sus padres. Por lo tanto, con el fin de atraer a los adolescentes a entrar y permanecer en la terapia, el terapeuta debe diseñar el proceso para que coincida con su ritmo, sus deseos y expectativas de cambio (Ronen \& Hamama, 2010).

Diversos estudios han empleado ejemplares caninos para trabajar como coterapeutas en el tratamiento de adolescentes con diferentes problemáticas, ya que a través de los animales, los adolescentes aprenden acerca de las interacciones sociales, las fronteras, la reciprocidad emocional y la responsabilidad. Los estudios han demostrado que los niños que son dueños de mascotas tienen más empatía por los demás, una alta autoestima y mejores habilidades sociales que los demás niños (Jalongo, Astorino \& Bomboy, 2004). El vínculo niño-animal es diferente a cualquier otro tipo de relación que un niño puede tener. Recientemente, Hamama, Hamama-Raz, Dagan, Greenfeld, Rubinstein \& Ben-Ezra (2011) emplearon perros en un programa de terapia asistida para trabajar con mujeres adolescentes que habían sufrido de abuso sexual. Los resultados mostraron que al término de la intervención las adolescentes presentaban una reducción en los síntomas de estrés postraumático y síntomas depresivos. Los autores concluyen que la terapia asistida por perros ofrece beneficios a los adolescentes que experimentan niveles elevados de ansiedad. En otro estudio se investigó si la tenencia de mascota y el apego del animal están relacionados con el autorreporte de soledad y apoyo social entre adolescentes (Black, 2012). Los resultados mostraron que los adolescentes que tienen mascotas refieren menos soledad que los adolescentes que no tienen mascota, por lo que el autor concluye que un animal de compañía puede aliviar la soledad que se experimenta en la adolescencia a través de la formación de vínculos de apego y la ampliación de las habilidades sociales.

Lo anterior indica que la terapia asistida por animales puede ser una intervención apropiada para tratar a los adolescentes con problemas emocionales, ya que los tipos de intervención que suelen emplearse se basan en las alabanzas y la retroalimentación sobre el desempeño, sin embargo, la interacción con un animal que afecta positivamente la autoestima y la autoimagen de los adolescentes, puede ayudarles a generar respuestas positivas para poder transferirlas posteriormente a otras personas y hacia el medio en general.
El objetivo de este estudio fue analizar los cambios en la autoestima y los componentes de las emociones de adolescentes que reciban terapia asistida por perros. Se espera que los adolescentes aumenten su autoestima y mejoren en el manejo de sus emociones al terminar las sesiones de terapia asistida por perros.

\section{Método}

\section{Participantes}

Aceptaron participar 15 adolescentes de edad promedio 18.7 (D.E. 1.16) años, 12 mujeres y 3 hombres. Sin embargo, no todos acudieron a la primera sesión y sólo 5 continuaron hasta el final, 4 mujeres y 1 hombre de edad promedio 18.8 (D.E. 1.3) años.

\section{Binomios para TAA}

Participaron 9 binomios voluntarios. Todos los perros cumplieron con los siguientes requisitos: cartilla de vacunación al corriente, desparasitados, limpieza de orejas, dientes y pelo. Todos los perros fueron evaluados respecto a su temperamento y obediencia básica, dos de los binomios cuentan con certificado para TAA. Los perros que participaron en la intervención tenían entre 1 y 5 años de edad, con un promedio de 2.34 años (D.E. $=1.02)$. La edad promedio de los manejadores fue de 31.6 años (D.E.=4.71), en su mayoría mujeres casadas.

\section{Instrumentos}

1. Carta de aceptación. Los pacientes firmaron una carta de consentimiento informado y los manejadores de los perros de terapia, un acuerdo de confidencialidad.

2. Escala de Autoestima de Rosenberg (Rosenberg, 1989). Esta es una escala tipo Likert diseñada para medir autoestima, la cual incluye 10 ítems con cuatro opciones de respuesta que van desde el nivel más bajo ("muy en desacuerdo") hasta el más alto ("muy de acuerdo"). Cinco de los ítems son inversos (1,3,4, 6 y 7). El contenido de éstos se centra en las dimensiones de autorrespeto y autoaceptación. Las puntuaciones van de 10 a 40, donde las más altas reflejan una mayor autoestima. Esta escala ha sido validada en 53 países, además de contar con traducción a más de 28 idiomas, mostrando valores alfa de Cronbach 
de entre 0.80 y 0.89 (Martín, Núñez, Navarro \& Grijalvo, 2007; Vázquez, Jiménez \& Vázquez, 2004).

3. Escala Triat Meta Mood Scale (TMMS-24). Es una escala diseñada para medir tres dimensiones clave de la inteligencia emocional, con 8 reactivos cada una de ellas: atención emocional, claridad de sentimientos y reparación emocional. Es una escala tipo Likert, con 5 opciones de respuesta, donde el 1 corresponde a "nada de acuerdo" y 5 corresponde a "totalmente de acuerdo". En este estudio se utilizó la versión adaptada al castellano, la cual ha mostrado una consistencia interna de 0.86 y 0.90 (Fernández-Berrocal et al., 2004).

4. Autoevaluación en cada sesión: Al inicio de cada sesión se les pedía a los pacientes que calificaran el manejo de sus emociones durante la semana anterior a la sesión, en una escala de 0 a 10 , siendo 0 nada de mejoría y 10 mucha mejoría.

5. Evaluación de beneficios de la TAA: Se les presentó a los pacientes una lista de palabras con los posibles beneficios sociales, cognitivos y emocionales de la terapia, y se les pidió que respondieran entre 0 y 10 , siendo 0 nada de beneficio y 10 muchísimo beneficio obtenido del taller.

\section{Materiales}

Para los perros: pelotas, cepillos, collares de nylon, correas, arnés, mochila, chaleco, kongs y galletas u otro premio.

Para el taller: láminas plastificadas de expresiones emocionales básicas de humanos y perros, y del lenguaje corporal canino.

\section{Procedimiento}

Una semana antes de iniciar el estudio, todos los participantes recibieron la información acerca del tipo de terapia a la que asistirían, firmaron la carta de aceptación y contestaron la Escala de Autoestima de Rosenberg y la escala TMMS-24. Posteriormente, los 5 participantes acudieron a 7 sesiones diseñadas como taller, una vez por semana, de 1 hora 30 minutos de duración cada una. En cada sesión participaron un mínimo de 3 binomios y todas las actividades del taller siempre estuvieron guiadas por el terapeuta.

El taller consistió en actividades diseñadas para aprender el manejo de las emociones, a través de la interacción con el perro. Las actividades de las sesiones quedaron de la siguiente manera: 1) Reconocimiento de las emociones básicas de alegría, tristeza, miedo, ira, asco y sorpresa, 2) Expresión de las emociones básicas, 3) Componentes de las emociones: percepción, comprensión y regulación, 4) Identificación correcta de las emociones en diversas situaciones futuras, 5) Pensamientos negativos y restructuración cognitiva, 6) Concepto de empatía y toma de decisiones, y 7) Aplicación de la Escala de Autoestima de Rosenberg, TMMS-24 y evaluación del taller.

\section{Análisis de datos}

Se realizaron análisis descriptivos y análisis de diferencia de grupos para alcanzar los objetivos del estudio. Al obtener las medidas de tendencia central de las evaluaciones antes y después del taller, se tomó la decisión de trabajar con casos ponderados para tener datos más exactos de la significancia estadística. Cada caso se ponderó por cuatro, conservando de esta manera un tamaño pequeño en los grupos y se utilizó la prueba no paramétrica: prueba de rangos de Wilcoxon. Todas las comparaciones se interpretarán como significativas con un error del $5 \%$ bilateral.

\section{Resultados}

\section{Autoestima y manejo emocional}

Los resultados que se presentan a continuación corresponden a los 5 participantes que concluyeron la intervención, 4 hombres y 1 mujer, de edad promedio 18.8 (D.E. 1.3) años. En la tabla 1 pueden observarse que los puntajes para atención emocional y reparación de las emociones son mayores en la evaluación post, sin embargo, el único dato significativo es la diferencia pre y post a la interacción con los perros en la atención emocional $(\mathrm{p}<.001)$. Estos resultados se ven influidos por el tamaño de muestra.

\section{Autoevaluación de las sesiones}

Para conocer la autoevaluación en el manejo emocional, se les pidió a los participantes que evaluaran su percepción del manejo y reconocimiento de sus emociones, así como también qué tanto consideraban que les había ayudado lo visto en la sesión anterior, todo esto en una escala de 0 a 10, siendo 0 nada de mejoría y 10 mucha mejoría. Los resultados se presentan en las siguientes gráficas. 


\section{Tabla 1.}

Puntuaciones de la escala de autoestima de Rosenberg y la escala TMMS-24 en la evaluación pre y post test.

\begin{tabular}{llllllllc}
\hline & \multicolumn{3}{c}{ Autoestima } & \multicolumn{2}{c}{ Atención emocional } & \multicolumn{2}{c}{ Claridad emocional } & \multicolumn{2}{l}{ Reparación de las emociones } \\
\hline & Pre & "Post & Pre & "Post & Pre & "Post & Pre & "Post \\
Media & 36.20 & 34.80 & 23.20 & 28.60 & 28.20 & 25.40 & 28.60 & 29.00 \\
Mediana & 37.00 & 37.00 & 23.00 & 28.00 & 29.00 & 25.00 & 30.00 & 31.00 \\
D.E. & 3.768 & 4.324 & 3.114 & 9.017 & 8.585 & 3.209 & 6.387 & 10.559 \\
Diferencia de grupos & $\mathrm{Z}=-1.236 ; \mathrm{p}=.216$ & $\mathrm{Z}=-1.490 ; \mathrm{p}=.136$ & $\mathrm{Z}=-.137 ; \mathrm{p}=.891$ & $\mathrm{Z}=-.271 ; \mathrm{p}=.786$ \\
Datos ponderados & $\mathrm{NS}$ & & $\mathrm{Z}=-3.330 ; \mathrm{p}=.001$ & $\mathrm{NS}$ & & $\mathrm{NS}$ & \\
\hline
\end{tabular}

D.E. $=$ Desviación estándar de la media.

NS $=$ No significativa .

$\mathrm{Z}=$ Valor estandarizado de la prueba de rangos de Wilcoxon.

Figura 1.

Promedio de las puntuaciones para la percepción del manejo de las emociones en las sesiones.

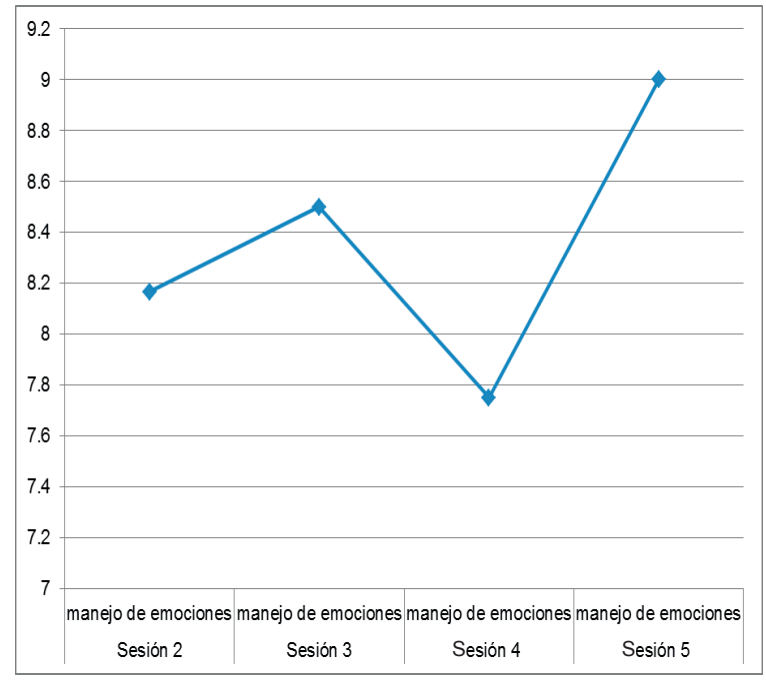

Figura 2.

Promedio de las puntuaciones para la percepción del reconocimiento de las emociones en las sesiones.

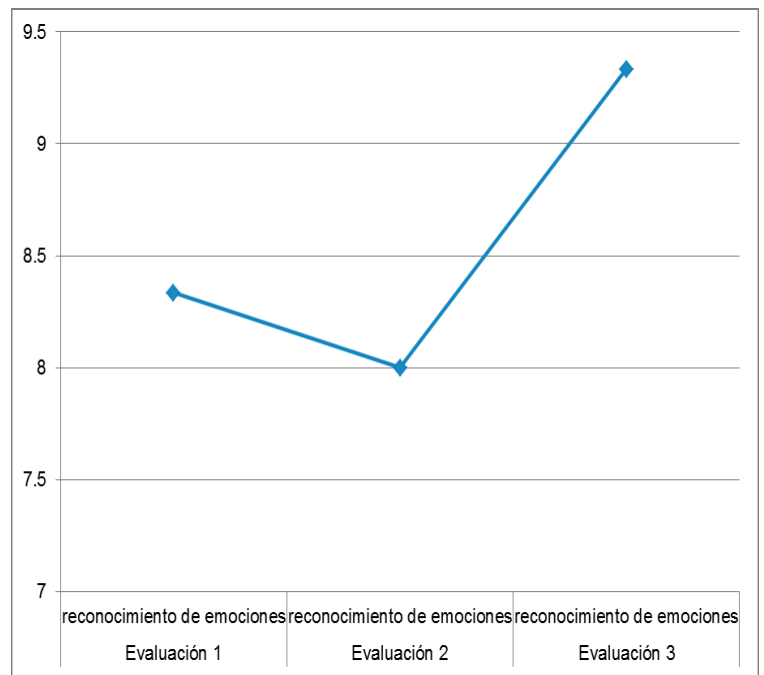


Figura 3.

Promedio de las puntuaciones para la percepción de la ayuda en el manejo de las emociones durante la semana.

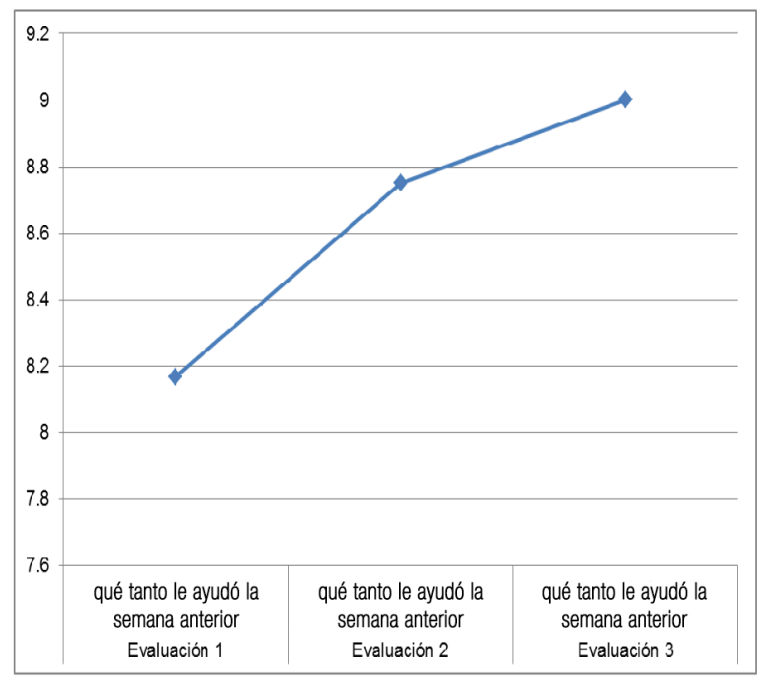

Como puede observarse, los participantes percibieron mejoría en su manejo de las emociones en las sesiones 3 y 5 ; en la sesión 4 hubo una disminución, se considera que el contenido de la sesión llevó a los participantes a dudar sobre el manejo de sus emociones, notándose una considerable mejoría en la última sesión (Figura 1). En lo que respecta al reconocimiento de las emociones, los participantes perciben una mejoría en este componente de las emociones, sobre todo al concluir el taller (Figura 2).

Finalmente, los participantes perciben que lo visto en la sesión anterior les ayudó en el manejo de sus emociones durante la semana, y esta percepción fue en aumento (Figura 3).

\section{Beneficios percibidos por los participantes}

La terapia asistida por animales está enfocada a lograr beneficios cognitivos, sociales, emocionales y personales. Se les presentó a los participantes una lista con estos posibles beneficios y se les pidió que respondieran entre 0 y 10 , siendo 0 nada de beneficio y 10 muchísimo beneficio obtenido del taller. Los resultados se muestran en la tabla 2, donde se observa que el mayor beneficio percibido fue en la capacidad de empatía.
Tabla 2.

Puntuaciones para los beneficios percibidos en lo cognitivo, social, emocional y personal de la terapia asistida por perros.

\begin{tabular}{llll}
\hline & Media & Mediana D.E. \\
\hline $\begin{array}{l}\text { Beneficios cognitivos (atención, com- } \\
\text { prensión, observación o memoria) }\end{array}$ & 9 & 2.16 \\
Beneficios sociales (trabajo en equipo) & 7.8 & 8 & 2.28 \\
Beneficios sociales (relaciones sociales) & 7.6 & 7 & 1.94 \\
Beneficios emocionales (capacidad & 8.8 & 10 & 1.78 \\
de empatía) & & & \\
Beneficios personales (autoestima) & 7.8 & 9 & 2.68 \\
\hline
\end{tabular}

\section{Relación de los participantes con sus propios perros}

Cuatro de los participantes tienen perro en casa, dos de ellos tienen 3, las edades de los perros van desde 5 hasta 13 años, con un promedio de 6.7 (D.E. $=1.34$ ) y todas son hembras. La mayoría consideraron que después del taller mejoró su relación con el perro, calificando esta mejoría entre 4 y 10 (en una escala de 0 a 10), lo mismo que su entendimiento de los perros, calificando esta mejoría entre 8 y 10 (en una escala de 0 a 10). Ninguno de los participantes reportó que sus hobbies incluyeran a sus perros; sus hobbies incluyeron actividades que implican conexión a internet, cine y lectura.

\section{Discusión}

En el presente estudio se analizaron los efectos de la terapia asistida por perros sobre el manejo de las emociones en adolescentes. El hallazgo principal de este estudio es que se encontró un aumento en los puntajes en uno de los componentes de las emociones, la atención emocional. Este resultado confirma parcialmente la hipótesis del estudio, ya que sólo se encontraron cambios en el componente de atención emocional, el cual se refiere a la capacidad de expresar y sentir las emociones de forma precisa, es decir, a la habilidad de percibir las emociones.

Este resultado coincide con los de otros estudios en donde se ha descrito que los adolescentes mejoran en algunos aspectos emocionales, como su autoestima, su capacidad de empatía y sentimientos de soledad (Black, 2012; Jalongo, Astorino \& Bomboy, 2004; Hamama 
et al., 2011). Este cambio en la atención emocional es importante, ya que la percepción adecuada de las emociones propias y de los demás es el primer requisito para lograr un manejo adecuado de las emociones. Las emociones se definen como las respuestas fisiológicas, conductuales y cognitivas ante un estímulo que las genera, por lo que la persona debe ser capaz de percibir adecuadamente todos los cambios en su cuerpo, su pensamiento y su conducta, para poder organizar y modular sus acciones. En cuanto a la autoestima, se debe tomar en cuenta que es una variable más estable, por lo que se considera que fue poco el tiempo para notar cambios significativos.

Otro de los cambios que se observó fue en los beneficios, específicamente en la capacidad de empatía, donde los adolescentes percibieron una mejoría en su capacidad para vincularse con otra persona y responder adecuadamente a las necesidades del otro, a compartir sus sentimientos e ideas de tal manera que logra que el otro se sienta muy bien con él. Cabe mencionar que este dato no fue significativo, pero indica la dirección de los datos y aporta información cualitativa acerca de las relaciones hombre-animal.

La terapia asistida por animales (TAA) se basa en los fuertes lazos humano-animal que son hasta ahora parcialmente comprendidos. Estos lazos son parte de nuestra historia evolutiva, en donde el ambiente natural juega un rol importante en la supervivencia y ha ayudado a dar forma a las diversas corrientes del desarrollo humano. En nuestra larga historia común con los animales, hay evidencia de una asociación entre los animales y la salud o bienestar, específicamente la interacción entre el ser humano y el perro se convirtió en una relación de beneficio (utilidad) y significativa (emocional) que ha durado por lo menos 10.000 años (Odendaal, 2000).

La TAA tiene lugar en diversos escenarios con diferentes grupos de edad y utiliza distintos tipos y razas de animales en función de quiénes sean los usuarios, el entorno y la estructura del programa. Con respecto a la TAA y los adolescentes, se ha encontrado que generalmente se utiliza en la terapia individual o con grupos pequeños, con el objetivo de garantizar los beneficios a largo plazo, donde la evidencia suele ser en gran parte cualitativa (Bryant, 1990). Lo que se ha observado con respecto a las emociones es que los adolescentes se sienten más cómodos hablando con los perros y de las emociones de los perros, ya que el perro acepta de forma incondicional, no juzga, es un catalizador para la enseñanza y aprendizaje, además de un distractor de los problemas (Rew, 2000).
La adolescencia es en sí misma un periodo estresante, confuso y difícil, en el que no todos los adolescentes comprenden los cambios físicos, cognitivos y emocionales que ocurren en ese periodo de la vida. Los problemas emocionales de los adolescentes pueden trabajarse con nuevas intervenciones como la TAA, ya que la interacción con los animales resulta un factor de motivación para que los adolescentes generen nuevas formas de vincularse con otras personas. Además podría utilizarse con adolescentes que presentan alguna enfermedad como ansiedad, depresión o que tienen conductas de riesgo, como abuso de drogas y conductas delictivas.

La principal limitación de este estudio es el tamaño de la muestra, sin embargo, de acuerdo con Burch (2000), los diseños de casos únicos o pocos sujetos pueden emplearse, pues tienen como objetivo demostrar una relación funcional entre un evento y un comportamiento específico del individuo. La evidencia que apoya este estudio abre nuevas propuestas de investigación sobre la naturaleza y calidad de las interacciones humano-animal

\section{Referencias}

Alonso, Y. (2000). ¿Los animales fomentan la salud mental? Un análisis preliminar. Revista de Psicología General y Aplicada, 53(4), 693-700.

Anzizu, L., Botella, L. \& Ferrer, J. (2001). Evaluación de una terapia asistida por animales de compañía (taac) en un colectivo de ancianos institucionalizados a partir del análisis del discurso de los usuarios. Psiquiatria.com, 5(3).

Black, K. (2012). The relationship between companion animals and loneliness among rural adolescents. Journal of Pediatric Nursing, 27(2), 103-112.

Bryant, B. (1990). The richness of the child-pet relationship: A consideration of both benefits and costs of pets to children. Anthrzoos, 3, 253-261.

Burch, M. (2000). Program evaluation and quality assurance in animal-assisted therapy. En A. Fine. (Ed.), Handbook on Animal Assisted Therapy: Theoretical Foundations and Guidelines for Practice. San Diego: Academic Press.

Damasio, A. (1994). Descartes' error: Emotion, reason, and the human brain. New York: Avon Books.

Denham, S. A., Blair, K. A., De Mulder, E., Levitas, J., Sawyer, K., Auerbach-Major, S., \& Queenan, P. (2003). Preschool emotional competence: Pathway to social competence. Child Development, 74(1), 238-256.

Eisenberg, N., Fabes, R. A., Guthrie, I. K., \& Reiser, M. (2000). Dispositional emotionality and regulation: Their role in predicting quality of social functioning. Journal of Personality and Social Psychology, 78(1), 136-157.

Elfenbein, H. A., Marsh, A. A., \& Ambady, N. (2002). Emotional intelligence and the recognition of emotion from facial expressions. En L. F. Barrett \& P. Salovey (Eds.), The wisdom in feeling: Psychological processes in emotional intelligence (pp. 37-59). New York: Guilford Press. 
Fernández-Berrocal, P., Extremera, N., \& Ramos, N. (2004). Validity and Reliability of the Spanish modified version of the trait metamood scale. Psychological Reports, 94, 751-755.

Fuentes, B., Gallego, J., Baza, S., \& Martín, N. (2012). Terapia asistida con perros como tratamiento rehabilitador en personas con esquizofrenia. $13^{\circ}$ Congreso Virtual de Psiquiatria.com. Interpsiquis 2012. http://www.interpsiquis.com

Gómez, L., Atehortua, C., \& Orozco, S. (2007). La influencia de las mascotas en la vida humana. Revista Colombiana de Ciencias Pecuarias, 20(3), 377-386.

González, M., \& Landero, R. (2011). Diferencias en estrés percibido, salud mental y física de acuerdo al tipo de relación humano-perro. Revista Colombiana de Psicología, 20(1), 75-86.

Guindon, M. H. (2002). Toward accountability in the use of the self-esteem construct. Journal of Counseling and Development, $80,204-214$.

Herbert, M. (2008). Adolescence. En M. Herbert (Ed.), The Blackwell companion to social work (pp. 368-376). (3rd ed.). Oxford, UK: Blackwell.

Hamama, L., Hamama-Raz, Y., Dagan, K., Greenfeld, H., Rubinstein, C., \& Ben-Ezra, M. (2011). A preliminary study of group intervention along with basic canine training among traumatized teenagers: A 3-month longitudinal study. Children and Youth Services Review, 33(10), 1975-1980.

Jalongo, M. R., Astorino, T., \& Bomboy, N. (2004). Canine visitors: The influence of therapy dogs on young children's learning and well-being in classrooms and hospitals. Early Childhood Education Journal, 32(1), 9-16.

Keltner, D., Moffit, T., \& Stouthamer-Loeber, M. (1995). Facial expression of emotion and psychopathology in adolescent boys. Journal of Abnormal Psychology, 104(4), 644-652.

Keltner, D., \& Haidt, J. (2001). Social functions of emotions. En T. J. Mayne \& G. A. Bonanno (Eds.), Emotions: Current issues and future directions. Emotions and social behavior (pp. 192-213). New York: Guilford Press.

Martín A., J., Núñez, J.L., Navarro, J.G., \& Grijalvo, F. (2007). The Rosenberg Self-Esteem Scale: Translation and validation in university students. The Spanish Journal of Psychology, 10(2), 58-67.

Odenadaal, J. (2000). Animal-assisted therapy: Magic or medicine. Journal of Psychosomatic Research, 49, 275-280.

Pearlin, L. I. (1989). The sociological study of stress. Journal of Health and Social. Behavior, 30, 241-256.

Rew, L. (2000). Friends and pets as companions: strategies for coping with loneliness among homeless youth. Journal of Child and Adolescent Psychiatric Nursing, 13(3), 125-132.

Ronen, T., \& Hamama, L. (2010). The role of cognitive developmental components in treating adolescence. Encyclopedia of Adolescence (pp. 1-10). New York: Springer.

Rosenberg, M. (1989). Society and the adolescent self-image (Revised edition). Middletown, CO: Wesleyan University Press.

Savage, C. R. (2002). The role of emotion in strategic behavior. En L. F. Barrett \& P. Salovey (Eds.), The wisdom in feeling (pp. 211-236). New York: Guilford Press.

Vázquez, M. A. J., Jiménez, G. B. R., \& Vázquez, M. J. R. (2004). Escala de Autoestima de Rosenberg: fiabilidad y validez en población clínica española. Apuntes de Psicología, 22, 247-250.

Vázquez, L., \& Lista, A. (2011). La terapia asistida con animales como parte de los programas de rehabilitación neuropsicológica. Psicologia.com, 15, 33-39. http://hdl.handle.net/10401/4385

Villalta, V., \& Ochoa, S. (2007). La terapia facilitada por animales de compañía como programa de rehabilitación adjunto para personas con diagnóstico de esquizofrenia crónica. Papeles del Psicólogo, 28(1), 49-56. 